\title{
SEVERITY OF ANXIETY AND WORK-RELATED OUTCOMES OF PATIENTS WITH ANXIETY DISORDERS
}

Steven R. Erickson, PharmD., ${ }^{1 *}$ Sally Guthrie, PharmD., ${ }^{1}$ Michelle VanEtten-Lee, Ph.D., ${ }^{2}$ Joseph Himle, Ph.D., ${ }^{3}$ Jody Hoffman, Ph.D., ${ }^{4}$ Susana F. Santos, PharmD., ${ }^{5}$ Amy S. Janeck Ph.D., ${ }^{6}$ Kara Zivin Ph.D., ${ }^{7,8}$ and James L. Abelson, M.D. ${ }^{7}$

\begin{abstract}
Background: This study examined associations between anxiety and workrelated outcomes in an anxiety disorders clinic population, examining botb pretreatment links and the impact of anxiety change over 12 weeks of treatment on work outcomes. Four validated instruments were used to also allow examination of their psychometric properties, with the goal of improving measurement of work-related quality of life in this population. Methods: Newly enrolled adult patients seeking treatment in a university-based anxiety clinic were administered four work performance measures: Work Limitations Questionnaire (WLQ), Work Productivity and Activity Impairment Questionnaire (WPAI), Endicott Work Productivity Scale (EWPS), and Functional Status Questionnaire Work Performance Scale (WPS). Anxiety severity was determined using the Beck Anxiety Inventory (BAI). The Clinical Global Impressions, Global Improvement Scale (CGI-I) was completed by patients to evaluate symptom change at a 12-week follow-up. Two severity groups (minimal/mild vs. moderate/severe, based on baseline BAI score) were compared to each other on work measures. Results: Eighty-one patients provided complete baseline data. Anxiety severity groups did not differ in job type, time on job, job satisfaction, or job choice. Patients with greater anxiety generally showed lower work performance on all instruments. Fob advancement was impaired for the moderate/severe group. The multi-item performance scales demonstrated better validity and internal consistency. The $W L Q$ and the WPAI detected change with symptom improvement. Conclusion: Level of work performance was generally associated with severity of anxiety. Of the instruments tested, the $W L Q$ and the
\end{abstract}

\footnotetext{
${ }^{1}$ College of Pharmacy, University of Michigan, Michigan ${ }^{2}$ Medical School, College of Literature, Science, and the Arts and Clinical Psychology, University of Michigan, Michigan ${ }^{3}$ Departments of Psychiatry and Social Work, University of Michigan, Michigan

${ }^{4}$ Ann Arbor Consultation Services

${ }^{5}$ Roche, Portugal

${ }^{6}$ Department of Psychology, University of British Columbia, British Columbia, Canada

${ }^{7}$ Department of Psychiatry, University of Michigan, Michigan

${ }^{8}$ Department of Veterans Affairs, National Serious Mental IIIness Treatment Research and Evaluation Center (SMITREC)

Contract grant sponsor: Upjohn Research Award Fund, College of Pharmacy, University of Michigan.
}

At the time of the study, Dr. Hoffman was a post-doctoral research fellow at the Department of Psychology, University of Michigan, Michigan.

At the time of the study, Dr. Santos was a visiting research student at the College of Pharmacy, University of Michigan, Michigan.

At the time of the study, Dr. Janeck was in the post-doctoral research training program of the Department of Psychiatry, University of Michigan, Michigan.

*Correspondence to: Steven R. Erickson, University of Michigan College of Pharmacy, 428 Church Street, Ann Arbor, MI 481091065. E-mail: serick@umich.edu

Received for publication 23 December 2008; Revised 21 August 2009; Accepted 22 August 2009

DOI 10.1002/da.20624

Published online 19 October 2009 in Wiley InterScience (www. interscience.wiley.com).

(C) 2009 Wiley-Liss, Inc. 
WPAI questionnaire demonstrated acceptable validity and internal reliability. Depression and Anxiety 26:1165-1171, 2009. (C) 2009 Wiley-Liss, Inc.

Key words: anxiety; work-outcomes; survey research; psychometric; productivity; absenteeism; presenteeism

\section{INTRODUCTION}

Anxiety disorders are common ${ }^{[1]}$ and costly, ${ }^{[2]}$ with lost work-productivity contributing substantially to their total costs and creating dependence on governmental support. ${ }^{[3-6]}$ They impact work performance by reducing employment opportunities, work functioning, and attendance. Pharmacological treatments can reduce symptoms and improve function, but also cause side effects that can undermine functional improvement. ${ }^{[7]}$ There is growing consensus that in order to optimize treatments, outcome studies should measure both symptomatic and functional recovery, including general quality of life and work performance. ${ }^{[8]}$

Work function studies in patients with panic disorder have documented high unemployment and low healthrelated quality of life; and even those with jobs report low work-productivity. ${ }^{[9,10]}$ Feeling disabled at work is associated with low "Quality of Well-Being." ${ }^{[11]}$ Most anxiety studies have focused on panic, so information on other anxiety disorders is limited, though anxiety diagnoses in general have been associated with impaired work performance. ${ }^{[12-14]}$ An epidemiological study ${ }^{[3,15]}$ also documented a narrowed range of employment opportunities, difficulty changing jobs, and restrictions in hours worked among people with anxiety disorders.

Integrating existing literature is difficult due to the wide range of instruments used, making comparisons across studies and across disorders difficult. ${ }^{[6]}$ Numerous work-function instruments have been validated over the past decade. ${ }^{[17,18]}$ Most can be used across disorders, but they vary in aspects of work function measured, and some may be better than others in identifying work impairment and tracking improvement for particular disorders.

This study examined self-reported work-productivity in adult patients with anxiety disorders, using four major instruments in wide-spread use at its inception (Work Limitations Questionnaire (WLQ), Endicott Work Productivity Scale (EWPS), Work Productivity and Activity Impairment Questionnaire (WPAI), and Work Performance Scale of the Functional Status Questionnaire (WPS)). Goal one was to examine the impact of anxiety severity on work-related outcomes in an anxiety specialty clinic population. Goal two was to examine psychometric properties (sensitivity, internal reliability, and construct validity) of the four instruments and to assess their relative suitability for use in this population.

\section{METHODS}

\section{DESIGN AND SAMPLE}

We measured self-reported health-related quality of life and workrelated outcomes for patients seeking anxiety treatment, prior to treatment and at 12-week follow-up. Patients seeking evaluation at the University of Michigan Anxiety Disorders Program between February 2002 and June 2005 were recruited and offered a \$20 incentive to participate. Entry criteria required presence of an anxiety disorder (generalized anxiety, panic, obsessive-compulsive disorder, or social phobia) in patients who were adult (18 years old) English speakers who worked outside the home at least $20 \mathrm{hr}$ per week for pay. Patients with schizophrenia, substance abuse, dementia, or suicidal ideation were excluded. The study was IRB approved and all subjects provided written, informed consent. Diagnoses were established on the basis of a two-hour clinical interview and consensus review by an interdisciplinary team.

\section{DATA COLLECTION}

Data were obtained from questionnaires and medical records. A work survey was mailed to all new patients as a single document containing the four work-productivity questionnaires (106 items), along with the consent document, a postage-free return envelope, and clinical questionnaires assessing symptom profiles and severity. Subjects were asked to return completed materials by mail before evaluation, but were also permitted to complete them at initial visit. Twelve weeks later, subjects with complete baseline data were mailed a follow-up packet containing only quality of life and work performance instruments.

\section{WORK MEASURES}

The WLQ ${ }^{[19]}$ covers a two-week time period with 25 items and 4 subscales: (1) "time management," (2) "physical demands," (3) "mental-interpersonal demands," and (3) "output demands." Subscale scores range from 0 (greatest impairment) to 100 (highest possible performance). The WPAI Questionnaire (WPAI, General Version) describes work, classroom, and activity impairment as a result of disease and/or treatment ${ }^{[20,21]}$ using four subscales: (1) "percent activity impairment," (2) "percent work time missed," (3) "percent impairment while working," and (4) "final overall work impairment." Scores range from 0 to $100 \%$ (complete impairment). The EWPS measures work-productivity ${ }^{[22]}$ in various job settings, including self-employment, using 25 items (0-4 scale) to quantify work-related behaviors, feelings, or attitudes over the past week. Item scores are summed to produce a total score with higher values reflecting greater impairment. The Functional Status Questionnaire WPS measures amount of work performed, time worked and breaks 
per shift, hours worked, accuracy and carefulness of work, healthrelated job changes, and fear of losing job due to health. ${ }^{[23]}$ It uses six items scored on a five-point scale, with low scores reflecting impairment.

Additional job-related questions were included on an investigator-formulated questionnaire to measure overall job satisfaction, length of time on the job, likelihood of choosing the same job again, and type of job (International Standard Classification of Occupations, 1988). Subjective ratings of "strongly agree" to "strongly disagree" were used to assess the influence of anxiety on job choice and job advancement. ${ }^{[2,25]}$ Number of work hours missed in the preceding week was included to assess absenteeism.

\section{SEVERITY AND CLINICAL CHANGE MEASURES}

The Beck Anxiety Inventory $(\mathrm{BAI})^{[26]}$ was obtained at baseline only, providing a general measure of anxiety severity applicable across diagnoses. Scores can range from 0 to 63 and are categorized as minimal (0-7), mild (8-15), moderate (16-25), and severe (26-63). Clinical improvement in disease severity over the 12-week follow-up was quantified using the Clinical Global Impressions, Global Improvement Scale (CGI-I), patient version. ${ }^{[27]}$

\section{ADDITIONAL MEASURES}

The SF-36 Health Survey Version 2 (SF-36) was used to assess health-related quality of life. ${ }^{[28]}$ It measures self-perceived concepts of physical functioning, role limitations due to physical health problems, bodily pain, general health, vitality, social function, role limitations due to emotional problems, and mental health. The "roleemotion" and "role-physical" scales include references to work, and together they function as a broad descriptor of role-functioning. The Sheehan Disability Scale (SDS) ${ }^{[29]}$ provided another general measure of role functioning (3 Likert measures of symptom interference with work, social life, and family/home life).

\section{ANALYSES}

The BAI was used to dichotomize patients into high (BAI scores of 16-63) and low (BAI 0-15) severity groups. The two groups were compared on demographic, job, and work-related data using $t$-tests and $\chi^{2}$ tests. Effect sizes for differences between groups were calculated using Cohen's $d$.

Patients were separately dichotomized using CCG-I scores (after 12 weeks of treatment) into those who perceived worsening or no change in their anxiety and those who perceived improvement. Separate paired $t$-tests were used for each group to determine whether the group improved significantly from baseline to follow-up on work-productivity measures. Effect sizes for differences in the change in scale scores between groups were calculated using Cohen's $d$.

Internal consistency of all multi-item subscales for the WLQ, EWPS, and WPS instruments was assessed using Cronbach's $\alpha$. Construct validity was assessed using Spearman's rank-order correlation, comparing work-productivity scale scores and the rolefunctioning scales of the SF-36 and the work-performance scale of the SDS.

Missing data from individual questionnaires were handled according to the recommendations of the developers of each questionnaire. Generally, if more than half of the item responses of a particular scale were missing, the scale score was not calculated. For all analyses, differences were considered significant at $P \leq 0.05$.

\section{RESULTS}

Because of personnel problems, active recruitment was underway during approximately half of the evaluation clinics conducted over the 3-year recruitment span. Because of manpower limitations, not all patients at each evaluation clinic could be approached for consent. We estimate that fewer than 300 of a potential pool of 717 patients were invited to participate, and a proportion of these did not meet age or diagnosis entry criteria. Of those eligible, 159 signed informed consent and 115 met the work requirement at the time of questionnaire completion. Eighty-one of these provided complete baseline data, and 50 provided both baseline and follow-up data.

\section{CHARACTERISTICS OF THE TWO SEVERITY GROUPS}

Of the 81 patients included in baseline analyses, 41 were categorized by the BAI as minimal-to-mild in severity and 40 were categorized as moderate-tosevere. Group demographic, illness, and health status data are presented in Table 1 . Groups did not differ in age, race, education, anxiety diagnosis, or physicalrelated health status. The high anxiety group contained more females and demonstrated lower mental-related health status, role physical, and role emotional scale scores, and greater Sheehan disability compared to the low anxiety group.

On work-related measures, the groups did not differ in the type of job, length of time on job, satisfaction with job, or job choice (Table 2). However, significantly more patients in the high anxiety group indicated that anxiety impaired their ability to advance in their job.

Group comparisons on work-performance scale scores are presented in Table 3. The high anxiety group was significantly more impaired than the low anxiety group on the WPS, the WPAI "percent impairment while at work," "overall work impairment," "percent activity impairment" subscales, and the WLQ "output demands," "physical demands," and "interpersonal demands" subscales. Effect sizes were generally moderate, ranging from 0.36 to 0.73 , except for a large anxiety severity effect (0.87) on WPAI-Percent Activity Impairment Due to Health. Three of the four WPAI scores had effect sizes over 0.7 , while the four WLQ scales effect sizes were 0.68 or less.

Of the 50 patients with both baseline and follow-up data, 38 improved and 12 did not change or worsened (Table 4). The group without symptom improvement showed no changes on any work performance measures. The symptomatically improved group showed mixed results. Many measures showed no change, but three of the four scales of the WPAI ("percent impairment while working," "final overall impairment due to health," and "percent activity impairment due to health") improved significantly in this group. They also improved 
TABLE 1. Comparison of demographic and anxiety variables between patients with minimal/mild anxiety to those with moderate/severe anxiety

\begin{tabular}{|c|c|c|c|}
\hline Variable & BAI minimal-to-mild anxiety $(n=41)$ & BAI moderate-to-severe anxiety $(n=40)$ & $P$-value \\
\hline Age-years, mean $(S D)$ & $37.5(12.2)$ & $34.2(9.8)$ & 0.17 \\
\hline \multicolumn{4}{|l|}{ Gender-frequency (\%) } \\
\hline Female & $20(48.8)$ & $30(75.0)$ & \multirow[t]{2}{*}{0.02} \\
\hline Male & $21(51.2)$ & $10(25.0)$ & \\
\hline \multicolumn{4}{|l|}{ Race-frequency (\%) } \\
\hline Caucasian & 39 & 39 & \multirow[t]{2}{*}{1.0} \\
\hline Minority & 1 & 0 & \\
\hline Education-years, mean $(S D)$ & $15.1(2.5)$ & $14.5(1.5)$ & 0.19 \\
\hline BAI Score, mean $(S D)$ & $9.2(4.5)$ & $26.9(7.6)$ & $<0.001$ \\
\hline \multicolumn{3}{|l|}{ Type of anxiety disorder } & \multirow[t]{12}{*}{0.3} \\
\hline OCD & $2(5.9)$ & $3(10.3)$ & \\
\hline Panic w/agor & $1(2.9)$ & $6(20.7)$ & \\
\hline Social phobia & $7(20.6)$ & $4(13.8)$ & \\
\hline GAD & $4(11.8)$ & $7(24.1)$ & \\
\hline Anxiety DO NOS & $2(5.9)$ & $0(0.0)$ & \\
\hline Specific phobia & $4(11.8)$ & $1(3.4)$ & \\
\hline Hypochondriasis & $1(2.9)$ & $1(3.4)$ & \\
\hline Body dysmorphic dis & $2(5.9)$ & $1(3.4)$ & \\
\hline Trichotillomania & $2(5.9)$ & $0(0.0)$ & \\
\hline Panic w/o agor & $4(11.8)$ & $3(10.3)$ & \\
\hline MDD & $5(14.7)$ & $3(10.3)$ & \\
\hline \multicolumn{4}{|l|}{ SF-36 scale scores } \\
\hline PCS & $56.9(8.8)$ & $53.3(9.9)$ & 0.1 \\
\hline MCS & $38.1(14.1)$ & $28.2(10.5)$ & 0.001 \\
\hline Role physical & $50.7(9.0)$ & $44.3(12.7)$ & 0.004 \\
\hline Role emotional & $40.3(12.8)$ & $31.0(12.6)$ & $<0.001$ \\
\hline \multicolumn{4}{|l|}{ Sheehan disability index } \\
\hline Work Life & $2.8(2.6)$ & $4.4(3.1)$ & 0.01 \\
\hline Social Life & $3.8(3.1)$ & $6.0(2.9)$ & 0.002 \\
\hline Home Life & $2.5(2.7)$ & $4.5(3.3)$ & 0.004 \\
\hline
\end{tabular}

Beck Anxiety Index: higher score indicates more severe anxiety. SF-36 Summary Scale Scores: higher score indicated better functioning. PCS, Physical Component Summary scale; MCS, Mental Component Summary Scale; Sheehan Disability Index: higher scale value indicates poorer performance, range from 0 , not impaired to 10 , very severely impaired.

TABLE 2. Comparison of work-related variables between patients with minimal/mild anxiety to those with moderate/ severe anxiety

\begin{tabular}{lcc}
\hline Variable & $\begin{array}{c}\text { Minimal-to-mild } \\
\text { anxiety }(n=41)\end{array}$ & $\begin{array}{c}\text { Moderate-to-severe } \\
\text { anxiety }(n=40)\end{array}$ \\
\hline Job description & & $5(12.2)$ \\
$\quad$ Secretarial & $2(4.9)$ & $0(0.0)$ \\
$\quad$ Agricultural & $1(2.4)$ & $2(4.9)$ \\
$\quad$ Skilled trades & $3(7.3)$ & $3(7.3)$ \\
$\quad$ Management & $5(12.2)$ & $5(12.2)$ \\
$\quad$ Sales & $1(2.4)$ & $12(29.3)$ \\
$\quad$ Professional & $20(48.8)$ & $13(34.1)$ \\
$\quad$ Other & $8(22.0)$ & $3.6(1.2)$ \\
Satisfaction with job & $3.6(1.4)$ & $3.1(1.2)$ \\
$\quad$ If you were looking for job, how likely would & & $4.7(4.7)$ \\
$\quad$ it be to take the same job you have now & $3.0(1.3)$ & $4.0(1.3)$ \\
Satisfaction with current job & $6.0(7.9)$ & $4.0(1.4)$ \\
Length of time on current job-years, mean $(S D)$ & $4.4(1.1)$ & $3.3(1.6)$ \\
Job choice influenced by anxiety** & $4.2(1.3)$ & 0.77 \\
If not for anxiety, I would have a different job** & $4.0(1.4)$ & 0.65 \\
Anxiety prevents job advancement* & & 0.40 \\
\hline
\end{tabular}

If looking for job, likelihood of taking same job: 1 , not likely at all; 5 , very likely. Satisfaction with job: 1 , very satisfied; 5 , very unsatisfied; ${ }^{* *}$ Scale ranges from 1 , strongly agree to 5 , strongly disagree. 
TABLE 3. Comparison of Work-Performance Questionnaires based on the BAI

\begin{tabular}{|c|c|c|c|c|}
\hline Work-Performance Questionnaire and scale scores & $\begin{array}{l}\text { Minimal-to-mild } \\
\text { anxiety }(n=41)\end{array}$ & $\begin{array}{l}\text { Moderate-to-severe } \\
\text { anxiety }(n=40)\end{array}$ & $P$-value & Effect size \\
\hline EWPS & $24.3(17.3)$ & $32.6(19.2)$ & 0.05 & -0.45 \\
\hline WPS score & $4.3(0.7)$ & $3.8(0.9)$ & 0.006 & 0.62 \\
\hline \multicolumn{5}{|c|}{ Work-Performance Activity Index - General Questionnaire } \\
\hline Percent work time missed due to health & $0.07(0.2)$ & $0.16(0.2)$ & 0.11 & -0.45 \\
\hline Percent impairment while at work due to health & $0.24(0.3)$ & $0.45(0.3)$ & 0.003 & -0.70 \\
\hline Final overall work impairment due to health & $0.28(0.3)$ & $0.50(0.3)$ & 0.003 & -0.73 \\
\hline Percent activity impairment due to health & $0.25(0.2)$ & $0.47(0.3)$ & 0.001 & -0.87 \\
\hline \multicolumn{5}{|l|}{ WLQ } \\
\hline Time management & $25.4(28.5)$ & $35.3(26.8)$ & 0.11 & -0.36 \\
\hline Output demands & $21.3(25.6)$ & $34.8(27.1)$ & 0.03 & -0.51 \\
\hline Physical demands & $11.8(24.9)$ & $30.3(32.6)$ & 0.005 & -0.64 \\
\hline Mental interpersonal demands & $27.1(21.8)$ & $37.4(20.9)$ & 0.03 & -0.48 \\
\hline
\end{tabular}

Endicott: 0, best performance; 4, poorest performance; Work-Performance Scale: 1, poorer performance; 5, best performance; WPAI: higher scale value indicates poorer performance, Work Limitations Questionnaire: higher scale value indicates poorer performance.

TABLE 4. Change in WPS scores based on patient perceived progress in illness over twelve weeks

\begin{tabular}{|c|c|c|c|c|c|}
\hline \multirow[b]{2}{*}{ Variable } & \multicolumn{2}{|c|}{ Perceived improved $(n=38)$} & \multicolumn{2}{|c|}{ Perceived no change or worsening $(n=12)$} & \multirow[b]{2}{*}{ Effect size } \\
\hline & Mean $(S D)$ change & $P$-value & Mean $(S D)$ Change & $P$-value & \\
\hline WPS & $0.14(0.78)$ & 0.28 & $-0.21(0.44)$ & 0.14 & 0.49 \\
\hline Endicott & $-3.13(13.9)$ & 0.17 & $6.45(12.5)$ & 0.12 & 0.71 \\
\hline WPAI percent work time missed & $0.04(0.19)$ & 0.26 & $0.01(0.05)$ & 0.44 & 0.19 \\
\hline WPAI percent impairment while working & $-0.18(0.32)$ & 0.002 & $0.01(0.22)$ & 0.89 & 0.69 \\
\hline WPAI final overall work impairment & $-0.15(0.33)$ & 0.01 & $0.02(0.21)$ & 0.72 & -0.61 \\
\hline WPAI percent activity impairment due to health & $-0.12(0.32)$ & 0.03 & $0.11(0.19)$ & 0.08 & -0.87 \\
\hline WLQ - time management & $-7.38(29.1)$ & 0.12 & $2.10(25.27)$ & 0.78 & -0.35 \\
\hline WLQ - output demands & $-10.0(24.6)$ & 0.01 & $8.75(18.60)$ & 0.13 & -0.86 \\
\hline WLQ - physical demands & $3.23(33.66)$ & 0.55 & $3.79(58.19)$ & 0.83 & -0.01 \\
\hline WLQ - mental interpersonal & $-12.02(22.4)$ & 0.002 & $8.80(17.48)$ & 0.11 & -1.03 \\
\hline
\end{tabular}

NA, could not be calculated.

significantly on two of the four WLQ subscales ("output demands" and "mental interpersonal"). Effect sizes for change score comparisons between groups were generally moderate (Table 4). Large effect sizes were observed for the WPAI-Percent Activity Impairment due to Health, the WLQ-Output Demands, and the WLQ MentalInterpersonal scale comparisons.

The instruments all had good internal consistency, with Cronbach's $\alpha$ as follows: $E W P S=0.95$, WPS $=0.82$, WLQ "time management" $=0.91$, "physical demands" $=0.97$, "mental interpersonal" $=0.92$, and "output demands" $=0.93$. Construct validity was assessed by correlating subscales of the work-productivity measures with the role-emotional and role-physical functioning scales of the SF-36, and with the SDS work scale (Table 5). The SF-36 role-emotional and the SDS work impairment scales were significantly correlated with all but one of the work-performance instruments and subscales. These correlations were moderate-to-good $(r=.4-.8)$. The WLQ "physical demands" subscale was significantly correlated with the SF-36 "role-emotional" score.

\section{DISCUSSION}

These data confirm that patients presenting for treatment in an academic anxiety disorders program have substantial work impairment and that greater work performance impairment is reported by those with higher anxiety severity. Similar relationships have been documented in other studies, for both depression and anxiety, examining work performance and attendance, using the WLQ, the Stanford Presenteeism Scale, and two single item scales measuring presenteeism days and inefficiency days. ${ }^{[12]}$ The presence of an anxiety disorder is also associated with less accomplished at work. ${ }^{[3]}$ Based on the WLQ, anxiety has a significant influence on interpersonal relationships at work as well as work output. The general measure WPAI confirms this association with significant findings based on Overall Work Impairment due to Health and Percent Impairment While at Work due to Health scale scores.

The WLQ and the WPAI demonstrated the most consistent psychometric properties of the four instruments examined. Both provide multiple subscales and most 


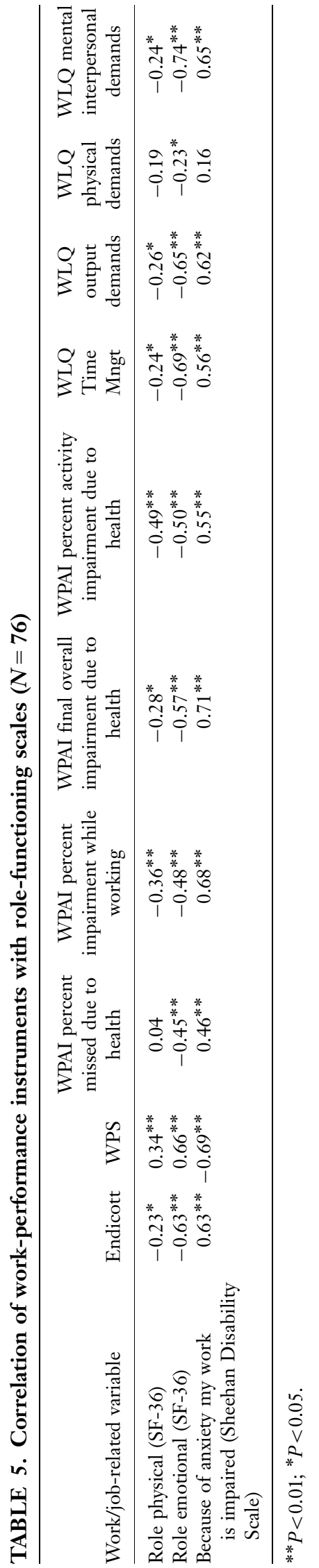

demonstrated acceptable validity. For example, there were significant differences on WLQ "output demands," "physical demands," and "mental interpersonal demands" scales based on comparison by anxiety severity, with better work performance associated with lower anxiety. Symptom severity effects on WPAI measured impairment have also been reported by others. ${ }^{[12]}$ Our moderate-to-large effect sizes for all comparisons based on severity support the validity of these scales.

We did not find anxiety group differences in WPAI "percent work time missed due to health" and the WLQ "physical demands" scale. This suggests that high anxiety may not lead to absences as often as physical illnesses, though impaired functioning while at work was clearly present in this group.

The WLQ demonstrated acceptable internal consistency. The WPAI uses single item scales so internal consistency is not relevant. Two of four WLQ scales ("output demands" and "mental interpersonal") and three of four WPAI scales (all but "percent time missed due to health") demonstrated sensitivity to changes in perceived clinical condition, with significant improvement in work function over the twelve week follow-up period in patients who perceived improvement in their disorder. Effect sizes for improvement group comparisons on symptom change scores were moderate to large, further confirming their ability to detect change.

The WPS and the EWPS did not function as effectively as the WLQ and the WPAI. Both showed good internal consistency, and the WPS was better able to distinguish work performance based on anxiety severity than the EWPS; but neither instrument was sensitive to symptom change in patients who improved, based on change within group comparisons.

Based on correlation analyses with role functioning and SDS-work scales, all instruments tested were valid in this anxious population. However, to be useful in comparative studies or clinical practice, instruments should be able to discriminate between various levels of disease severity. The WPAI and WLQ scales demonstrated this ability; the EWPS and WPS did not. The WPAI provides a good general assessment of time missed from work and percent impairment using only 6 questions, which tracked both anxiety severity and improvement with treatment. It provides a good, brief instrument when a general measure is sufficient. When more detailed, domain specific work performance data is needed, the 25 item WLQ can be used, alone or in conjunction with the WPAI., to provide a more comprehensive picture of work functioning.

\section{LIMITATIONS}

We enrolled only a subset of patients seeking treatment and do not have good data to document how representative these were of our total clinic population, though we have no evidence of any systematically biasing factors in our recruitment efforts. We also recruited only 
from an academic anxiety specialty clinic. Further work is needed to determine the generalizability of our results.

We included only patients with anxiety disorders and did not document the presence of physical illness. We therefore cannot evaluate the impact of concurrent physical illness on work performance in these anxious patients, and cannot compare them to patients with other psychiatric disorders. We also only included patients who had jobs outside their homes. Since anxiety patients have high rates of nonparticipation in the work force, ${ }^{[3]}$ we likely underestimated the true impact of anxiety on work function.

Test-retest reliability was not assessed as part of the psychometric analysis. We were focused on the association of clinical outcomes with work performance, so we used a 12 week follow-up period to capture significant clinical improvement, and predicted clinically-related change in work performance measures. Assessment of test-retest stability would require a different design and a much shorter reassessment period.

\section{CONCLUSION}

Work performance was measured using four work performance instruments in a clinic sample of anxiety disorder patients. Greater illness severity was associated with greater impairment in work-performance. Work performance improved with symptom improvement. For this population, we recommend the WPAI as a brief, general measure of work function and the WLQ when a more detailed, domain specific instrument is needed, as both showed good psychometric performance, sensitivity to illness severity, and sensitivity to change with symptomatic improvement.

Acknowledgments. No conflict of interest is present for any author.

\section{REFERENCES}

1. Kessler RC, Berglund P, Demler O, et al. Lifetime prevalence and age-of-onset distributions of DSM-IV disorders in the national Comorbidity Survey Replication. Arch Gen Psychiatry 2005;62:593-602.

2. Greenberg PE, Sisitsky T, Kessler RC, et al. The economic burden of anxiety disorders in the 1990s. J Clin Psychiatry 1999;60:427-435

3. Waghorn G, Chant D, White P, Whiteford H. Disability, employment and work performance among people with ICD-10 anxiety disorders. Aust N Z J Psychiatry 2005;39:55-66.

4. Henderson M, Glozier N, Elliott KH. Long term sickness absence. BMJ 2005;330:802-803.

5. Hardy G, Woods D, Wall TD. The impact of psychological distress on absence from work. J Appl Psychol 2003;88:306-314.

6. Waghorn G, Chant D. Work performance among Australians with depression and anxiety disorders. J Nerv Ment Dis 2006;194:898-904.

7. Haslam C, Brown S, Atkinson S, Haslam RA. Patients' experiences of medication for anxiety and depression: effects on working life. Fam Pract 2004;21:204-212.
8. Hollifield M, Katon W, Skipper B, et al. Panic disorder and quality of life: variables predictive of functional impairment. Am J Psychiatry 1997;154:766-772.

9. Ettigi P, Meyerhoff AS, Chirban JT, et al. The quality of life and employment in panic disorder. J Nerv Ment Dis 1997;185:368-372.

10. Katerndahl DA, Realini JP. Quality of life and panic-related work disability in subjects with infrequent panic and panic disorder. J Clin Psychiatry 1997;58:153-158.

11. Rubin HC, Rapaport MH, Levine B, et al. Quality of well being in panic disorder: the assessment of psychiatric and general disability. J Affect Disord 2000;27:217-221.

12. Sanderson K, Tilse E, Nicholson J, Oldenburg B, Graves N. Which presenteeism measures are more sensitive to depression and anxiety? J Affect Disord 2007;101:65-74.

13. Sanderson K, Andrews G. Common mental disorders in the workforce: recent findings from descriptive and social epidemiology. Can J Psychiatry 2006;51:63-75.

14. Kessler RC, Greenberg PE, Mickelson KD, et al. The effects of chronic medical conditions on work loss and work cutback. J Occup Environ Med 2001;43:218-225.

15. Waghorn GR, Chant DC. Employment restrictions among persons with ICD-10 anxiety disorders: characteristics from a population survey. J Anxiety Disord 2005;19:642-657.

16. Mendlowicz MV, Stein MB. Quality of life in individuals with anxiety disorders. Am J Psychiatry 2000;157:669-682.

17. Lofland JH, Pizzi L, Frick KD. A review of health-related workplace productivivty loss instruments. Pharmacoeconomics 2004;22:164-184.

18. Prasad M, Wahlquist P, Shikiar R, Shih YCT. A review of selfreported instruments measuring health-related work productivity. Pharmacoeconomics 2004;22:225-244.

19. Lerner D, Amick BC, Rogers WH, et al. The work limitations questionnaire. Med Care 2001;39:72-85.

20. Reilly MC, Zbrozek AS, Dukes EM. The validity and reproducibility of a work productivity and activity impairment instrument. Pharmacoeconomics 1993;4:353-365.

21. Reilly MC, Tanner A, Meltzer EO. Work, classroom and activity impairment instruments. Validation studies in allergic rhinitis. Clin Drug Investig 1996;11:278-288.

22. Endicott J, Nee J. Endicott Work Productivity Scale (EWPS): a new measure to assess treatment effects. Psychopharmacol Bull 1997;33:13-16.

23. Jette AM, Davies AR, Cleary PD, et al. The functional status questionnaire: reliability and validity when used in primary care. J Gen Intern Med 1986;1:143-149.

24. Landy FJ, Farr JL. The Measurement of Work Performance. Methods, Theory, and Applications. New York: Academic Press; 1983.

25. Quinones MA, Ford JK, Teachout MS. The relationship between work experience and job performance: a conceptual and metaanalytic review. Pers Psychol 1995;48:887-910.

26. Beck AT, Steer RA. Beck Anxiety Inventory Manual. San Antonio, TX: The Psychological Corporation Harcourt Brace and Company; 1993.

27. Guy W. ECDEU Assessment Manual for PsychopharmacologyRevised (DHEW Publ No ADM 76-338). Rockville, MD, U.S. Department of Health, Education, and Welfare, Public Health Service, Alcohol, Drug Abuse, and Mental Health Administration, NIMH Psychopharmacology Research Branch, Division of Extramural Research programs; 1976:218-222.

28. Ware JE, et al. SF-36 Health Survey: Manual and Interpretation Guide. Boston, MA: Health Institute, New England Medical Center; 1993.

29. Sheehan DV. The Anxiety Disease. New York: Schribner's; 1983. 